\title{
Erratum: Extended Drude Model for Intraband-Transition-Induced Optical Nonlinearity [Phys. Rev. Applied 11, 064062 (2019)]
}

\author{
Heng Wang, ${ }^{1}$ Kang Du, ${ }^{1}$ Chuhao Jiang, ${ }^{1}$ Zhiqiang Yang, ${ }^{1}$ Lixia Ren, ${ }^{1}$ Wending Zhang,,${ }^{1, \dagger}$ \\ Soo Jin Chua, ${ }^{1,2,3}$ and Ting Mei® ${ }^{1, *}$ \\ ${ }^{1}$ MOE Key Laboratory of Material Physics and Chemistry under Extraordinary Conditions, and Shaanxi Key \\ Laboratory of Optical Information Technology, School of Physical Science and Technology, Northwestern \\ Polytechnical University, Xi'an 710129, China \\ ${ }^{2}$ Department of Electrical and Computer Engineering, National University of Singapore, 4 Engineering Drive 3, \\ Singapore 117583 \\ ${ }^{3}$ LEES Program, Singapore-MIT Alliance for Research \& Technology (SMART), 1 CREATE Way, \#10-01 \\ CREATE Tower, Singapore 138602
}

(Received 28 July 2020; published 16 September 2020)

DOI: 10.1103/PhysRevApplied.14.039901

The degeneracy factors in Eq. (7) are known to be equal to the number of distinct permutations of the applied field frequencies. According to the multinomial theorem, for cross modulation, the degeneracy factors of $\chi^{(3)}, \chi^{(5)}$, and $\chi^{(7)}$ are calculated as $3 ! /(1 ! \times 1 ! \times 1 ! \times 0 !)=6,5 ! /(2 ! \times 2 ! \times 1 ! \times 0 !)=30$, and $7 ! /(3 ! \times 3 ! \times 1 ! \times 0 !)=140$, respectively. Thus, Eq. (7) should be corrected to

$$
\begin{aligned}
\varepsilon_{r}= & 1+\chi^{(1)}(\omega)+6 \chi^{(3)}\left(\omega: \omega^{\prime},-\omega^{\prime}, \omega\right)\left|E_{p}\left(\omega^{\prime}\right)\right|^{2} \\
& +30 \chi^{(5)}\left(\omega: \omega^{\prime},-\omega^{\prime}, \omega^{\prime},-\omega^{\prime}, \omega\right)\left|E_{p}\left(\omega^{\prime}\right)\right|^{4}+\cdots,
\end{aligned}
$$

where the degeneracy factor of $\chi^{(5)}$ is 30 instead of 20 in the original paper.

The plot of $\chi^{(5)}$ in Fig. 4(d) is corrected as shown below:

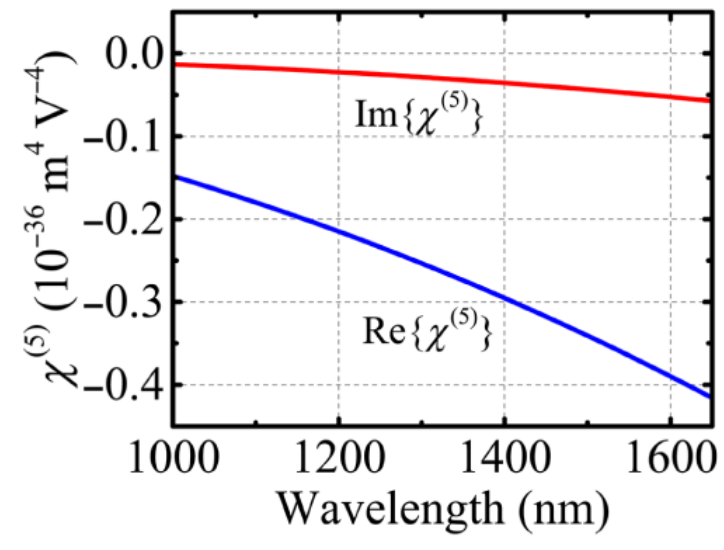

Corrected Fig. 4(d).

In the optical nonlinearity fitting in Fig. S2 within the Supplemental Material, the degeneracy factors of $\chi^{(5)}$ and $\chi^{(7)}$ should be 30 and 140, respectively, instead of 20 and 42 in the original paper. Thus, the corrected figures in Fig. S2 are shown below:

*ting.mei@ieee.org

†zhangwd@nwpu.edu.cn 
(b3)

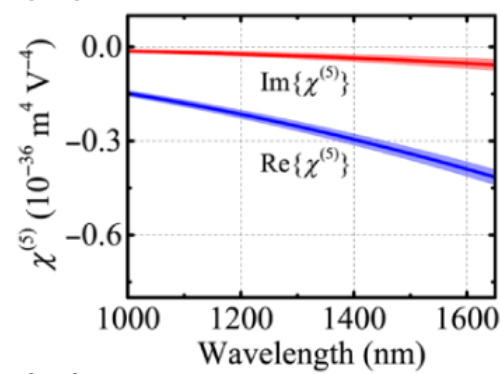

(c3)

(c4)
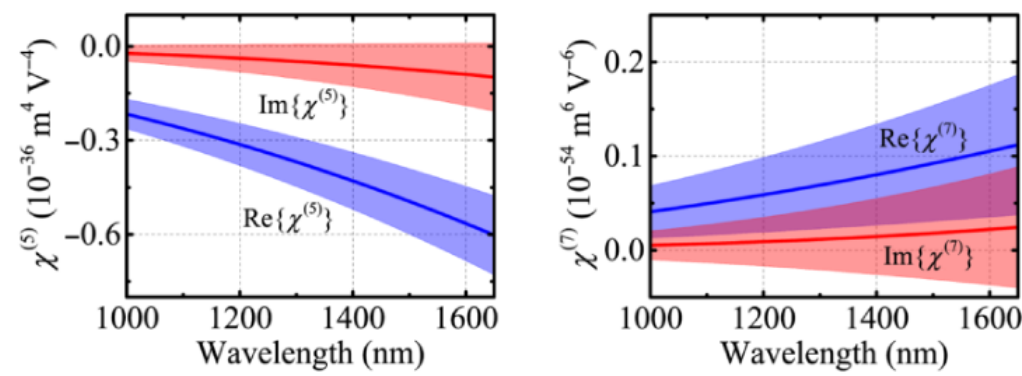

Corrected Figs. S2b3, S2c3, and S2c4.

These corrections do not affect the conclusion of the paper.

We thank Lin Cheng, Akbar Safari and Rasoul Alaee from the University of Ottawa for reminding us of this issue. 